\title{
Herb-drug interactions: A review and study based on assessment of clinical case reports in literature
}

\author{
K.J. Gohil, J .A. Patel*
}

\begin{abstract}
(2)
Department of Pharmacology, Maliba Pharmacy College, Gopal Vidyanagar, Tarsadi, Bardoli, Surat,

*D epartment of Pharmacology,

Institute of Pharmacy, Nirma

U niversity of Science and

Technology, Ahmedabad, India

Received: 10.7.2006

Revised: 16.2.2007

Accepted: 10.6.2007

Correspondence to: Jagruti A. Patel,

E-mail: jagrutiap@gmail.com
\end{abstract}

\begin{abstract}
O bjective: To conduct a systematic review of literature on interactions between conventional drugs and various herbs.

Materials and Methods: We carried out a literature survey to assess published herbdrug interaction information in clinical case reports and case series to check the report reliabilities.

Results: From 133 cases of suspected interactions, $67 \%$ cases were classified as possible interactions, $27 \%$ cases were unable to be evaluated and only $6 \%$ of the cases were well-documented. St. John's wort was the most common herb involved (37 cases) in drug interactions. Warfarin was the most common drug (34 cases) interacting with various herbs.

Conclusion: Herb-drug interactions are a stark reality today. Hence, proper reporting of cases, careful vigilance, evidence-based appraisal and constantly updated reviews of such herb-drug interactions are very important to promote systematic research.
\end{abstract}

KEY WORDS: Case series, clinical assessment, clinical case reports, herb-drug interactions

\section{Introduction}

Millions of people today use herbal therapies along with prescription and nonprescription medications. Although considered natural, many of these herbal therapies can interact with other medications, causing either potentially dangerous side effects and / or reduced benefits from the medications. Currently, there is very little information published on herb-drug interactions whilst the use of herbs is progressively growing across the world.

The size of the worldwide market of herbal medicines is estimated to be around US $\$ 80$ billion to US $\$ 100$ billion and this market is expected to reach US $\$ 2500$ billion by the year $2010 .{ }^{[1-2]}$ In the West, the demand for herbal drugs has reached a new high in recent years. Since 1999, the global market for herbal supplements exceeded US \$15 billion, with a US \$ 7 billion market in Europe, US $\$ 2.4$ billion in Japan, US $\$ 2.7$ in the rest of Asia and US $\$ 3$ billion in North America. ${ }^{[3]}$ The results of a nationwide survey indicated a marked increase in the number of individuals using alternative therapies between 1990 and 1997 estimating total out-of-pocket expenditures for alternative therapies at $\$ 27$ billion. ${ }^{[4]}$

India is very rich in natural resources and the knowledge of traditional medicine and the use of plants as a source of medicine is an innate and very important component of the healthcare system. The Indian system of medicine has identified 1,500 medicinal plants of which 500 are commonly used. ${ }^{[2]}$ It is estimated that there are over 7800 medicinal drugmanufacturing units in India, which are estimated to consume about 2000 tons of herbs annually. ${ }^{[5]}$ According to a recent estimate of the World Health Organization (WHO), $70-80 \%$ of the world population especially in developing countries, relies on traditional medicine, mostly plant drugs for their primary healthcare needs. ${ }^{[2,6]}$

As the use of herbs is getting popular, interactions between herbs and allopathic drugs need to be addressed and reviewed properly. Certain herbal supplements can cause potentially dangerous side effects when taken with prescription drugs. The use of alternative therapy is mostly not supervised by physicians or alternative therapy practitioners resulting in increased harm to patients, especially if they are using herbal and prescription medicines that have latent interactions. These interactions can go unnoticed until a patient is hurt or a serious life-threatening event has occurred.

Despite the urgent need for information, very little is known about herb-drug interactions unfortunately as experimental data in this field is limited and case reports and case series are rare. ${ }^{[7-8]}$

\section{Objective}

The aim of our present study was to conduct a review of literature on interactions between conventional drugs and various herbs based on descriptions of the clinical consequences, case series and case reports. In addition, we 
also wanted to assess the quality of the data generated for the study of herb-drug interactions.

\section{Materials and Methods}

A literature survey was carried out to compile information about herb-drug interactions. As per the guidelines for the use of electronic and internet media, ${ }^{[9-10]}$ a high quality and reliable medical information from the internet was retrieved only from the Health-on-Net (HON) conduct-certified and accredited websites like Entrez PubMed [Medline], CAM-PubMed, Allied and complementary medicine database, Natural medicine comprehensive database, Embase and Cochrane library. The databases were searched from 1986-2006 using the search terms "herb-drug interactions, side effects, herb toxicities, adverse drug reactions, herbal medicine, herbal medicinal, prescriptions, case reports, case series, clinical trials and clinical assessments". Non-English language citations were excluded. An extensive review of the literature identified reported herb-drug interactions with clinical significance, many of which were in the form of case reports and limited clinical observations. The nature of the findings and probability of interactions were then abstracted and compiled in a final report [Table 1]. The probability of interactions was evaluated on a 10 point scoring scale reported earlier, ${ }^{[8]}$ where each of the case reports received one point for inclusion of any of the following ten evaluating parameters:

- Adequate patient history.

- Concurrent diseases, conditions.

- Documentation of concomitant medications.

- Adequate description of interactions.

- Exclusion of obvious alternative explanations.

- Complete chronology.

- Reasonable time sequence of drug administration to adverse event.

- Adequate description of adverse event.

- Cessation of event on stopping the drug.

- Recurrence of event with a re-challenge.

This probability scale, though not validated, served only as a guide to assess whether the reports of herb-drug interactions contained reliable information or not. The sum total of all these parameters was calculated and referred to as report reliability scores as per the criteria mentioned below. This report reliability score was further validated by a third party, i.e., two independent raters unaware of the study protocol.

a. A score of 0-3 suggested that the case contains insufficient information (unable to be evaluated).

b. A score of 4-7 implied some evidence for an interaction (possible) and

c. A score of 8-10 indicated a well-documented report with reliable evidence for interaction (likely).

\section{Results}

The search of all listed sources produced 133 cases of suspected interactions. All interactions were tabulated and categorized by herb, drug, other medications, signs or symptoms of interaction, mechanism and report reliability scores [Table 1]. $67 \%$ of the cases $(89 / 133)$ were classified as possible interactions, $27 \%(36 / 133)$ were considered to contain insufficient information to evaluate the probability of an interaction (Unable to be evaluated) and $6 \%(08 / 133)$ as welldocumented (likely) interactions. St. J ohn's wort was the herb most commonly implicated in interactions with various drugs (37 cases) namely digoxin (13 cases), clopidogrel (06 cases), indinavir (08 cases), irinotecan (05 cases), antipsychotics (04 cases) and tacrolimus (01 case). Other herbs most commonly implicated in herb-drug interactions were ginkgo biloba (27 cases), kava-kava (08 cases), ginger (06 cases) and Panax ginseng ( 05 cases). The anticoagulant drug, warfarin was found to be the most common drug interacting with various herbs (34 cases) of which 22 cases were classified as 'unable to be evaluated' interactions, 10 cases were 'possible' interactions and 2 cases were classified as 'well-documented' or 'likely' interactions.

\section{Discussion}

The results of our search point towards the fact that herb-drug interactions certainly do occur and are much more common than we would like to believe, contrary to the popular belief that Nature is always safe. In many cases, mechanisms and causality are uncertain or unpredictable; in addition, inadequate information and under-reporting make it very difficult to determine whether herb-drug interactions have occurred or not. In the past, very few case reports related to herb-drug interactions were reported and many reactions could only be explained theoretically. Recently, however, there are several reported cases of possible herb-drug interactions with the increased use of herbal medications. ${ }^{[11-14]}$

An earlier survey of literature for reports of herb-drug interactions presented 108 cases of suspected interactions out of which $68.5 \%$ were reported as unable to be evaluated, $18.5 \%$ as possible and $13 \%$ as well documented cases. ${ }^{[8]}$ In this study, 133 new cases of suspected herb-drug interactions were identified of which $27 \%$ cases $(36 / 133)$ were unable to be evaluated, $67 \%$ cases $(89 / 133)$ were described as possible and only $06 \%$ cases $(08 / 133)$ emerged as well documented interactions.

In the present study, St. J ohn's Wort was the most common herb implicated in various drug interactions and warfarin was the most common drug interacting with various herbs. ${ }^{[8,15-18]}$ A previous study reported 85 interaction cases of St. John's Wort of which 54 cases were with the drug cyclosporin. Other drug categories mentioned were oral contraceptives (12 cases), antidepressants (09 cases), warfarin (07 cases) and one case each with theophylline, phenprocoumon and loperamide interacting with St. J ohn's Wort..$^{[8]}$ The present study identified 37 cases of interactions for St. J ohn's Wort with digoxin (13 cases), clopidogrel (06 cases), indinavir (08 cases), irinotecan (05), antipsychotics (03), tacrolimus (01 case) and with an anesthetic (01 case). This makes St. J ohn's Wort a somewhat risky proposition when combined with drugs in the categories mentioned above.

As with conventional medicines, herbal medicines interact with drugs in two general ways: pharmacokinetically and pharmacodynamically. ${ }^{[14]}$ Pharmacokinetic interactions result in alterations of the drug's or natural medicine's absorption, distribution, metabolism or elimination. These interactions affect drug action by quantitative alterations, either increasing or decreasing the amount of drug available to have an effect. 


\section{Table 1}

Assessment of clinical case reports on herb-drug interactions

\begin{tabular}{|c|c|c|c|c|c|c|c|}
\hline $\begin{array}{l}\text { Case } \\
\text { No. }\end{array}$ & $\begin{array}{c}\text { Patient- descrip- } \\
\text { tion }\end{array}$ & Herb & Drug & $\begin{array}{l}\text { Other } \\
\text { medica- } \\
\text { tions }\end{array}$ & $\begin{array}{l}\text { Symptom(s) of } \\
\text { interaction }\end{array}$ & Mechanism & $\begin{array}{c}\text { Report-reliability } \\
\text { score }\end{array}$ \\
\hline $1-13$ & $\begin{array}{c}\text { St. John's Wort } \\
\text { used by } 13 \\
\text { volunteers for two } \\
\text { weeks. }{ }^{[32]}\end{array}$ & $\begin{array}{l}\text { St. John's } \\
\text { Wort } \\
\text { Hypericum } \\
\text { perforatum } \\
\text { (Depression) }\end{array}$ & Digoxin & None & $\begin{array}{c}\text { Reduction in } \\
\text { serum digoxin } \\
\text { level by } 18-25 \%\end{array}$ & $\begin{array}{l}\text { Induction of } \\
\text { P-glycopro- } \\
\text { tein by St. } \\
\text { John's Wort }\end{array}$ & $\begin{array}{c}\text { Possible } \\
0+1+1+1+1+0+ \\
1+1+0+0=6\end{array}$ \\
\hline $22-27$ & $\begin{array}{l}\text { Case series } 06 \\
\text { healthy volun- } \\
\text { teers. }{ }^{[35]}\end{array}$ & $\begin{array}{l}\text { St. John's } \\
\text { Wort } \\
\text { Hypericum } \\
\text { perforatum } \\
\text { (Depression) }\end{array}$ & $\begin{array}{l}\text { Clopidogrel } \\
\text { (Plavix) }\end{array}$ & None & $\begin{array}{l}\text { Decline in platelet } \\
\text { aggregation }\end{array}$ & $\begin{array}{l}\text { Induction of } \\
\text { Cyp450 }\end{array}$ & $\begin{array}{c}\text { Possible } \\
1+1+1+0+0+ \\
0+0+1+0+0=4\end{array}$ \\
\hline 28 & $\begin{array}{l}20 \text { year-old col- } \\
\text { lege student with } \\
\text { major depressive } \\
\text { episode. } .^{[50]}\end{array}$ & $\begin{array}{l}\text { St. John's } \\
\text { Wort } \\
\text { Hypericum } \\
\text { perforatum } \\
\text { (Depression) }\end{array}$ & $\begin{array}{l}\text { Clonaz- } \\
\text { epam }\end{array}$ & Lithium & $\begin{array}{c}\text { Extreme } \\
\text { agitation, irritabil- } \\
\text { ity, pressured } \\
\text { speech, anxiety }\end{array}$ & Not known & $\begin{array}{c}\text { Possible } \\
1+1+1+0+0+0 \\
+0+1+0+0=4\end{array}$ \\
\hline 29 & $\begin{array}{l}\text { A } 51 \text { year-old } \\
\text { female with his- } \\
\text { tory of psychotic } \\
\text { mania.. }{ }^{50]}\end{array}$ & $\begin{array}{l}\text { St. John's } \\
\text { Wort } \\
\text { Hypericum } \\
\text { perforatum } \\
\text { (Depression) }\end{array}$ & Haloperidol & Lithium & $\begin{array}{l}\text { Bizarre behavior, } \\
\text { Excessive motor } \\
\text { activity and disor- } \\
\text { ganized speech. }\end{array}$ & Not known & $\begin{array}{c}\text { Possible } \\
1+1+1+0+0+0 \\
+0+1+0+0=4\end{array}$ \\
\hline 30 & Not known. ${ }^{[107]}$ & $\begin{array}{l}\text { St. John's } \\
\text { Wort } \\
\text { Hypericum } \\
\text { perforatum } \\
\text { (Depression) }\end{array}$ & Olanzapin & & $\begin{array}{c}\text { Olanzapine level } \\
\text { is increased by } \\
300 \%\end{array}$ & $\begin{array}{l}\text { Inhibition of } \\
\text { CYP1A2 }\end{array}$ & $\begin{array}{l}\text { Unevaluable } \\
0+1+0+1+0+0 \\
+0+1+0+0=3\end{array}$ \\
\hline 32 & $\begin{array}{l}\text { A kidney trans- } \\
\text { plant patient. }{ }^{[109]}\end{array}$ & $\begin{array}{l}\text { St. John's } \\
\text { Wort } \\
\text { Hypericum } \\
\text { perforatum } \\
\text { (Depression) }\end{array}$ & Tacrolimus & None & $\begin{array}{l}\text { Lower blood } \\
\text { levels of drug }\end{array}$ & $\begin{array}{l}\text { Induction } \\
\text { of CYP3A4 } \\
\text { system }\end{array}$ & $\begin{array}{c}\text { Possible } \\
1+1+0+0+1+0+0+ \\
1+1+1+0=6\end{array}$ \\
\hline 33-37. & $\begin{array}{l}\text { Five cancer pa- } \\
\text { tients: two men, } \\
\text { three women with } \\
\text { a median age of } \\
58 \text { years }^{[110]}\end{array}$ & $\begin{array}{l}\text { St John's Wort } \\
\text { Hypericum } \\
\text { perforatum } \\
\text { (Depression) }\end{array}$ & Irinotecan & None & $\begin{array}{l}\text { Reduction in } \\
\text { serum irinotecan } \\
\text { level results in } \\
\text { decreased side } \\
\text { effects of drug }\end{array}$ & $\begin{array}{l}\text { Induction of } \\
\text { Cyp450 }\end{array}$ & $\begin{array}{c}\text { Possible } \\
1+1+0+0+1+0+0+ \\
1+1+1+0=6\end{array}$ \\
\hline 38 & $\begin{array}{c}33 \text { year-old } \\
\text { woman with } \\
\text { bilateral subdural } \\
\text { hematomas }^{[20,55]}\end{array}$ & Ginkgo biloba & $\begin{array}{l}\text { Acetamino- } \\
\text { phen }\end{array}$ & $\begin{array}{l}\text { Ergota- } \\
\text { mine } \\
\text { caffeine }\end{array}$ & $\begin{array}{l}\text { Increased bleed- } \\
\text { ing time up to } 15 \\
\text { min }\end{array}$ & $\begin{array}{c}\text { Herb has } \\
\text { antiplatelet } \\
\text { Activity }\end{array}$ & $\begin{array}{c}\text { Likely } \\
1+1+1+1+0+1 \\
+1+1+1+0=8\end{array}$ \\
\hline 39 & No information ${ }^{[57]}$ & $\begin{array}{l}\text { Ginkgo biloba } \\
\text { (Circulatory } \\
\text { disorders) }\end{array}$ & Ibuprofen & None & $\begin{array}{c}\text { Fatal } \\
\text { intracerebral } \\
\text { hemorrhage }\end{array}$ & $\begin{array}{l}\text { Herb has } \\
\text { Antiplatelet } \\
\text { activity }\end{array}$ & $\begin{array}{l}\text { Unevaluable } \\
\begin{array}{c}0+1+0+1+1+0+0 \\
+0+0+0=3\end{array}\end{array}$ \\
\hline $40-61$ & $\begin{array}{c}\text { Case series } n=22 \\
\text { Healthy sub- } \\
\text { jects. }^{[77]}\end{array}$ & Ginkgo biloba & Nifedipine & None. & $\begin{array}{l}\text { Increase in mean } \\
\text { plasma level of } \\
\text { Nifedipine }\end{array}$ & $\begin{array}{l}\text { Inhibition of } \\
\text { CYP3A4 }\end{array}$ & $\begin{array}{c}\text { Possible } \\
0+1+1+1+1 \\
+0+0+1+1+0=6\end{array}$ \\
\hline
\end{tabular}


Table 1 contd...

\begin{tabular}{|c|c|c|c|c|c|c|c|}
\hline $\begin{array}{l}\text { Case } \\
\text { No. }\end{array}$ & $\begin{array}{l}\text { Patient- descrip- } \\
\text { tion }\end{array}$ & Herb & Drug & $\begin{array}{c}\text { Other } \\
\text { medica- } \\
\text { tions }\end{array}$ & $\begin{array}{l}\text { Symptom(s) of } \\
\text { interaction }\end{array}$ & Mechanism & $\begin{array}{c}\text { Report-reliability } \\
\text { score }\end{array}$ \\
\hline $62-63$. & $\begin{array}{l}\text { Two elderly } \\
\text { epilepsy } \\
\text { patients }^{[111]}\end{array}$ & $\begin{array}{l}\text { Ginkgo biloba } \\
\text { (Circulatory } \\
\text { disorders) }\end{array}$ & $\begin{array}{c}\text { Sodium } \\
\text { valproate }\end{array}$ & None & $\begin{array}{l}3 \text { to } 4 \text { seizures } \\
\text { within } 2 \text { weeks }\end{array}$ & Not known & $\begin{array}{c}\text { Likely } \\
1+1+0+1+1+1 \\
+1+1+1+0=8\end{array}$ \\
\hline 64 & $\begin{array}{l}34 \text { year-old male } \\
\text { undergoing lapa- } \\
\text { roscopic chole- } \\
\text { cystectomy }{ }^{[12-113]}\end{array}$ & Ginkgo biloba & $\begin{array}{c}\text { No } \\
\text { medication } \\
\text { stated }\end{array}$ & None. & $\begin{array}{l}\text { Increase in } \\
\text { postoperative } \\
\text { bleeding }\end{array}$ & $\begin{array}{c}\text { Inhibit } \\
\text { platelet } \\
\text { Activating } \\
\text { factor }\end{array}$ & $\begin{array}{c}\text { Possible } \\
1+1+0+1+1+0+ \\
0+1+0+0=5\end{array}$ \\
\hline 65 & $\begin{array}{l}\text { One man-no } \\
\text { other } \\
\text { information }{ }^{[65,78]}\end{array}$ & $\begin{array}{l}\text { Green tea } \\
\text { Camellia } \\
\text { sinensis }\end{array}$ & Warfarin & None & $\begin{array}{l}\text { Thickening of } \\
\text { blood }\end{array}$ & $\begin{array}{c}\text { Herb has } \\
\text { antagonistic } \\
\text { Effect }\end{array}$ & $\begin{array}{c}\text { Possible } \\
0+1+0+1+1+0 \\
+1+0+1+0=5\end{array}$ \\
\hline 66 & $\begin{array}{c}\text { A } 44 \text { year-old } \\
\text { man with a } \\
\text { mechanical heart } \\
\text { valve }^{[65,78]}\end{array}$ & $\begin{array}{l}\text { Green tea } \\
\text { Camellia } \\
\text { sinensis }\end{array}$ & Warfarin & None & $\begin{array}{c}\text { Decreased INR to } \\
1.37 \text { from } 3.79\end{array}$ & $\begin{array}{l}\text { Herb has } \\
\text { antagonistic } \\
\text { effect due to } \\
\text { presence } \\
\text { of vitamin } \mathrm{K} \\
\text { in herb }\end{array}$ & $\begin{array}{c}\text { Possible } \\
1+1+0+1+0+0 \\
+1+1+0+0+0=5\end{array}$ \\
\hline 67 & $\begin{array}{l}\text { A } 72 \text { year-old } \\
\text { lady }{ }^{[65]}\end{array}$ & $\begin{array}{c}\text { Coenzyme Q } \\
\text { (ubiquinone } \\
\text { or ubidecare- } \\
\text { none) }\end{array}$ & Warfarin & & $\begin{array}{l}\text { Decreased } \\
\text { response to } \\
\text { warfarin }\end{array}$ & $\begin{array}{c}\text { Herb } \\
\text { possesses } \\
\text { procoagulant } \\
\text { property }\end{array}$ & $\begin{array}{c}\text { Possible } \\
1+1+0+1+0+0 \\
0+1+1+0=5\end{array}$ \\
\hline 68 & $\begin{array}{l}\text { A } 68 \text { year-old } \\
\text { male with history } \\
\text { of pulmonary and } \\
\text { cerebro vascular } \\
\text { disease } \\
{[65]}\end{array}$ & $\begin{array}{l}\text { Coenzyme Q } \\
\text { (ubiquinone or } \\
\text { ubidecare- } \\
\text { none }\end{array}$ & & & $\begin{array}{c}\text { Decreased INR to } \\
1.31 \text { from } 2-3\end{array}$ & $\begin{array}{c}\text { Herb } \\
\text { possesses } \\
\text { procoagulant } \\
\text { property }\end{array}$ & $\begin{array}{c}\text { Likely } \\
1+1+0+1+1+1 \\
+1+1+1+0=8\end{array}$ \\
\hline 69 & $\begin{array}{c}\text { A } 67 \text { year-old } \\
\text { female with atrial } \\
\text { fibrillation }^{[76]}\end{array}$ & $\begin{array}{l}\text { Boldo } \\
\text { Peumus } \\
\text { boldus } \\
\text { Fenugreek }\end{array}$ & & Metopro- & INR increased & Not known & $\begin{array}{c}\text { Possible } \\
1+1+1+0+0+0+1 \\
+0+0+0=4\end{array}$ \\
\hline 70 & No information ${ }^{[79]}$ & $\begin{array}{l}\text { Quilinggao } \\
\text { (Chinese } \\
\text { herbal product }\end{array}$ & & None & Bleeding episode & Synergism & $\begin{array}{l}\text { Unevaluable } \\
0+1+0+1+0+0 \\
+0+1+0+0=3\end{array}$ \\
\hline 71 & $\begin{array}{c}\text { No information } \\
\text { provided }^{[80]}\end{array}$ & $\begin{array}{l}\text { Lycium } \\
\text { barbarum } \\
\text { (Chinese } \\
\text { herbal tea) }\end{array}$ & Warfarin & None & $\begin{array}{l}\text { Interfere with the } \\
\text { effect of warfarin }\end{array}$ & Not known & $\begin{array}{c}\text { Unevaluable } \\
0\end{array}$ \\
\hline 72 & ${ }_{\text {information }}^{\text {No1-82] }}$ & $\begin{array}{c}\text { Saw palmetto } \\
\text { Serenoa } \\
\text { repens }\end{array}$ & Warfarin & None. & Increased INR & Not known & $\begin{array}{c}\text { Unevaluable } \\
2\end{array}$ \\
\hline 73 & $\begin{array}{l}\text { A } 36 \text { year-old } \\
\text { male with } \\
\text { depression }^{[86]}\end{array}$ & $\begin{array}{c}\text { Ayahusca } \\
\text { Banisteriopsis } \\
\text { caapi }\end{array}$ & Fluoxetine & None & $\begin{array}{l}\text { Tremors, shiver- } \\
\text { ing, sweating, } \\
\text { severe nausea } \\
\text { and vomiting }\end{array}$ & Not known & $\begin{array}{c}\text { Unevaluable } \\
1+1+0+0+0+0+ \\
0+0+0+0=2\end{array}$ \\
\hline $74-75$. & $\begin{array}{c}55 \text { year-old } \\
\text { female and } \\
\text { another } 49 \\
\text { year-old patient. } \\
\text { No other } \\
\text { information }^{[87]}\end{array}$ & $\begin{array}{c}\text { Celery } \\
\text { Apium } \\
\text { graveolens }\end{array}$ & Thyroxin & None & Decreased T4 & Not known & $\begin{array}{c}\text { Possible } \\
1+1+0+0+0+0+ \\
1+1+0+0=4\end{array}$ \\
\hline 76 & $\begin{array}{c}60 \text { year-old male } \\
\text { with hypertension } \\
\text { and } \mathrm{CHF} .{ }^{[88]}\end{array}$ & $\begin{array}{c}\text { Fennel fruit } \\
\text { Foeniculam } \\
\text { vulgar }\end{array}$ & Enalapril & None. & $\begin{array}{l}\text { Patient was free } \\
\text { from cough after } \\
\text { chewing fennel }\end{array}$ & Not known & $\begin{array}{c}\text { Possible } \\
1+1+1+1+1+1 \\
+0+0+0+0=6\end{array}$ \\
\hline
\end{tabular}


Table 1 contd...

\begin{tabular}{|c|c|c|c|c|c|c|c|}
\hline $\begin{array}{c}\text { Case } \\
\text { No. }\end{array}$ & $\begin{array}{c}\text { Patient- descrip- } \\
\text { tion }\end{array}$ & Herb & Drug & $\begin{array}{l}\text { Other } \\
\text { medica- } \\
\text { tions }\end{array}$ & $\begin{array}{l}\text { Symptom(s) of } \\
\text { interaction }\end{array}$ & Mechanism & $\begin{array}{c}\text { Report-reliability } \\
\text { score }\end{array}$ \\
\hline 77 & $\begin{array}{l}57 \text { year-old male } \\
\text { with type } 2 \\
\text { diabetes }^{[89]}\end{array}$ & $\begin{array}{l}\text { Prickly pear } \\
\text { Opuntia } \\
\text { streptacantha }\end{array}$ & $\begin{array}{l}\text { Oral hypo- } \\
\text { glycemic } \\
\text { agent }\end{array}$ & None & $\begin{array}{c}\text { Mean fasting } \\
\text { glucose level } \\
\text { increased up to } \\
205 \mathrm{mg} / \mathrm{dl}\end{array}$ & Not known & $\begin{array}{c}\text { Possible } \\
1+1+1+1+0+0 \\
+1+1+0=0=6\end{array}$ \\
\hline 78 & $\begin{array}{c}\text { Healthy } \\
\text { volunteers }^{[90]}\end{array}$ & $\begin{array}{c}\text { Garlic } \\
\text { Allium sativum } \\
\text { (Hypercholes- } \\
\text { terolemia) }\end{array}$ & Saquinavir & None & $\begin{array}{l}\text { Significantly } \\
\text { reduced plasma } \\
\text { Saquinavir } \\
\text { concentration }\end{array}$ & Unknown & $\begin{array}{c}\text { Unevaluable } \\
\begin{array}{c}0+1+1+0+1+0+0 \\
+0+0+0=3\end{array}\end{array}$ \\
\hline $79-84$ & $\begin{array}{l}\text { Six Danish } \\
\text { rheumatoid arthri- } \\
\text { tis patients } \\
{[91]}\end{array}$ & $\begin{array}{l}\text { Ginger } \\
\text { Zingiber } \\
\text { officinale }\end{array}$ & NSAIDS & None & $\begin{array}{c}\text { No symptomatic } \\
\text { relief }\end{array}$ & Not known & $\begin{array}{c}\text { Possible } \\
0+1+1+1+0+0+1 \\
+1+0+0=5\end{array}$ \\
\hline 85 & No information ${ }^{[92]}$ & $\begin{array}{l}\text { Licorice } \\
\text { Glycyrrhiza } \\
\text { glabra }\end{array}$ & Laxative & None & Potassium loss & $\begin{array}{c}\text { Additive } \\
\text { effect }\end{array}$ & $\begin{array}{l}\text { Unevaluable } \\
0+1+0+1+0+ \\
0+0+0+0+0=2\end{array}$ \\
\hline 86 & No information ${ }^{[93]}$ & $\begin{array}{l}\text { Licorice } \\
\text { Glycyrrhiza } \\
\text { glabra }\end{array}$ & Sennoside & None & $\begin{array}{l}\text { Myoclonus due } \\
\text { to metabolic } \\
\text { alkalosis }\end{array}$ & Not known & $\begin{array}{c}\text { Unevaluable } \\
2\end{array}$ \\
\hline 87 & No information ${ }^{[94]}$ & $\begin{array}{l}\text { Soybeans } \\
\text { Glycine max }\end{array}$ & Warfarin & None & $\begin{array}{l}\text { Decline in INR } \\
\text { reduced } \\
\text { anticoagulation }\end{array}$ & Not known & $\begin{array}{c}\text { Possible } \\
\begin{array}{c}0+1+0+1+1+1 \\
=4\end{array}\end{array}$ \\
\hline 88 & $\begin{array}{c}\text { A } 59 \text { year-old } \\
\text { Caucasian } \\
\text { female received } \\
\text { a cadaveric renal } \\
\text { transplant } 16 \\
\text { years before this } \\
\text { admission }{ }^{[95]}\end{array}$ & $\begin{array}{c}\text { Alfalfa } \\
\text { Medicago } \\
\text { sativa }\end{array}$ & $\begin{array}{c}\text { Immuno- } \\
\text { suppres- } \\
\text { sants. } \\
\text { Azathioprin } \\
\text { Cyclosporin }\end{array}$ & None & $\begin{array}{l}\text { She began to } \\
\text { reject the trans- } \\
\text { planted kidney. }\end{array}$ & Not known & $\begin{array}{c}\text { Possible } \\
1+1+1+1+1+1 \\
+0+1+0+0=7\end{array}$ \\
\hline $89-90$ & $\begin{array}{l}\text { Two Patients of } \\
\text { epilepsy no other } \\
\text { information }^{[96]}\end{array}$ & $\begin{array}{l}\text { Shankha- } \\
\text { pushpi } \\
\text { Evolvulus } \\
\text { alsinoides }\end{array}$ & Phe & & $\begin{array}{l}\text { Loss of seizure } \\
\text { control }\end{array}$ & Not known & $\begin{array}{c}\text { Possible } \\
0+1+1+1+0+0+1 \\
+1+0+0=5\end{array}$ \\
\hline 91 & $\begin{array}{c}\text { A } 47 \text { year-old } \\
\operatorname{man}^{[64]}\end{array}$ & $\begin{array}{c}\text { Ginseng } \\
\text { Panax ginseng }\end{array}$ & & $\begin{array}{l}\text { Diltiazem } \\
\text { Nitro- } \\
\text { glycerin } \\
\text { Salsalate }\end{array}$ & $\begin{array}{l}\text { INR declined } \\
\text { to } 1.5\end{array}$ & Not known & $\begin{array}{c}\text { Possible } \\
1+1+1+0+0+0+ \\
1+1+1+0=6\end{array}$ \\
\hline 92 & $\begin{array}{l}\text { A post-meno- } \\
\text { pausal woman }{ }^{[114]}\end{array}$ & $\begin{array}{c}\text { Ginseng } \\
\text { (Topical) } \\
\text { Panax ginseng }\end{array}$ & $\begin{array}{l}\text { Hormone } \\
\text { therapy }\end{array}$ & None & $\begin{array}{c}\text { Mastalgia and } \\
\text { vaginal bleeding }\end{array}$ & $\begin{array}{c}\text { Herb shows } \\
\text { Estrogen-like } \\
\text { Effect }\end{array}$ & $\begin{array}{c}\text { Possible } \\
0+1+1+1+0+0+0 \\
1+0+0=4\end{array}$ \\
\hline 93 & $\begin{array}{c}\text { A } 39 \text { yearr-old fe- } \\
\text { male experienced } \\
\text { menometrorrha- } \\
\text { gia }^{[115]}\end{array}$ & $\begin{array}{c}\text { Ginseng } \\
\text { Panax ginseng }\end{array}$ & $\begin{array}{l}\text { Caffeine } \\
\text { and nico- } \\
\text { tine }\end{array}$ & None & Sinus tachycardia & Not known & $\begin{array}{c}\text { Possible } \\
1+1+0+1+1+1+ \\
1+0+1+0=7\end{array}$ \\
\hline 94 & $\begin{array}{l}\text { A patient with } \\
\text { depression }{ }^{[116]}\end{array}$ & $\begin{array}{l}\text { Ginseng } \\
\text { Panax ginseng }\end{array}$ & Lithium & $\begin{array}{l}\text { Amitrip- } \\
\text { tyline }\end{array}$ & Maniac episode & Not known & $\begin{array}{c}\text { Possible } \\
1++1+1+0+0+1 \\
+0+0+0+0=4\end{array}$ \\
\hline 95 & $\begin{array}{l}\text { A woman with } \\
\text { major depres- } \\
\text { sion. } .^{[117]}\end{array}$ & $\begin{array}{c}\text { Ginseng } \\
\text { Panax ginseng }\end{array}$ & $\begin{array}{l}\text { Clomip- } \\
\text { ramine }\end{array}$ & $\begin{array}{l}\text { Haloperi- } \\
\text { dol }\end{array}$ & Maniac episode & Not known & $\begin{array}{c}\text { Possible } \\
1+1+1+0+0+0\end{array}$ \\
\hline $96-99$ & $\begin{array}{l}\text { Case series } \\
\qquad(n=4)^{[60]}\end{array}$ & $\begin{array}{c}\text { Kava } \\
\text { Piper } \\
\text { methysticum }\end{array}$ & Levodopa & None & Dyskinesia & Not known & $\begin{array}{l}\text { Unevaluable } \\
0+0+0+1+0+ \\
0+1+1+0+0=3\end{array}$ \\
\hline 100 & $\begin{array}{c}\text { A } 29 \text { year-old } \\
\operatorname{man}^{[118]}\end{array}$ & $\begin{array}{c}\text { Kava } \\
\text { Piper } \\
\text { methysticum }\end{array}$ & $\begin{array}{c}\text { Gaurana } \\
\text { (caffeine) }\end{array}$ & Gingko & $\begin{array}{l}\text { Myoglobinuria } \\
\text { Rhabdomyolysis } \\
\text { Severe muscle } \\
\text { pain, dark urine } \\
\text { and elevated } \\
\text { creatine kinase }\end{array}$ & Not known & $\begin{array}{c}\text { Possible } \\
1+0+1+1+0+0+ \\
0+1+0+0=4\end{array}$ \\
\hline
\end{tabular}


Table 1 contd...

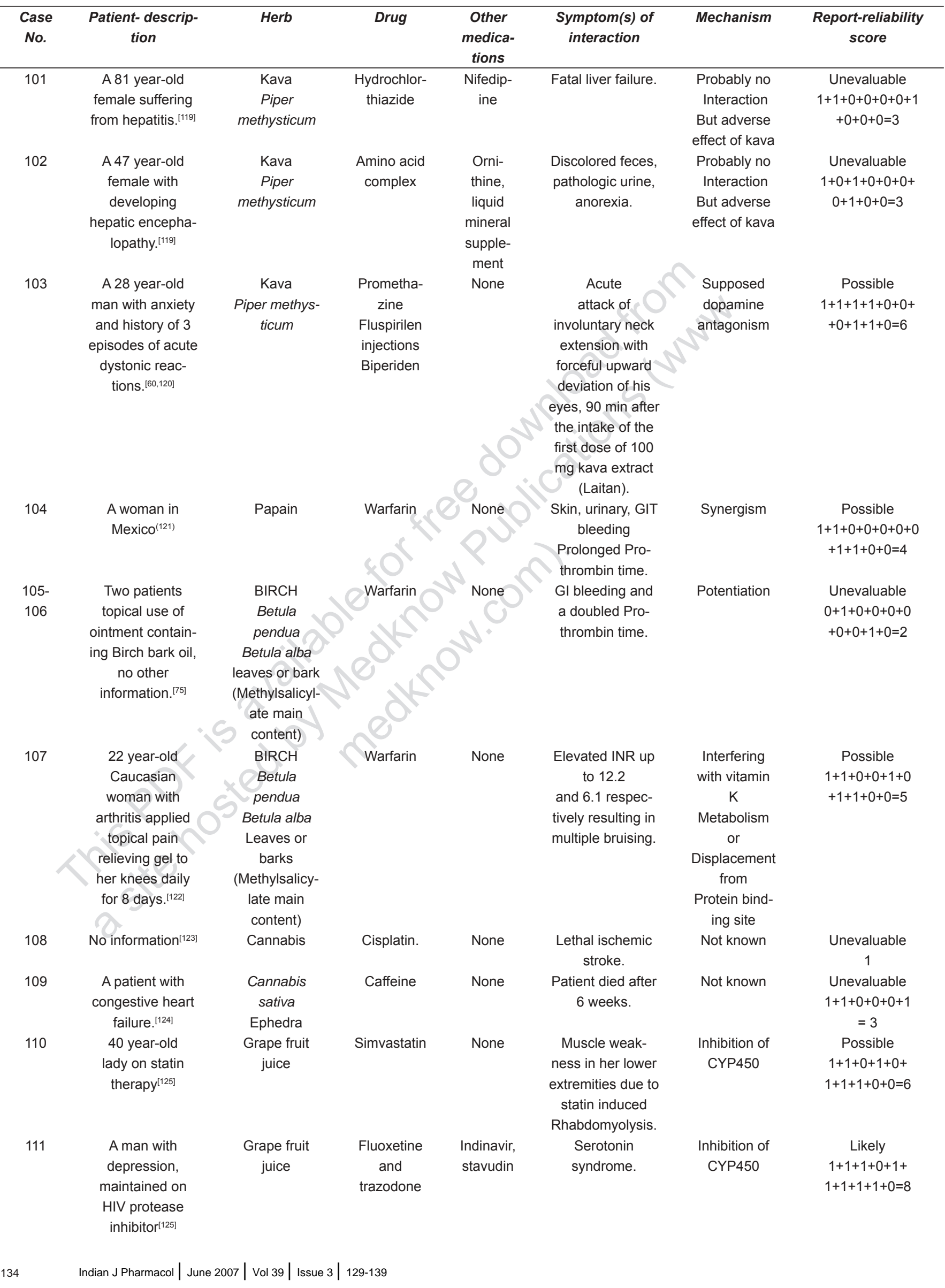


Table 1 contd...

\begin{tabular}{|c|c|c|c|c|c|c|c|}
\hline $\begin{array}{l}\text { Case } \\
\text { No. }\end{array}$ & $\begin{array}{l}\text { Patient- descrip- } \\
\text { tion }\end{array}$ & Herb & Drug & $\begin{array}{c}\text { Other } \\
\text { medica- } \\
\text { tions }\end{array}$ & $\begin{array}{l}\text { Symptom(s) of } \\
\text { interaction }\end{array}$ & Mechanism & $\begin{array}{c}\text { Report-reliability } \\
\text { score }\end{array}$ \\
\hline 112 & $\begin{array}{l}\text { A diabetic } \\
\text { woman }^{[126]}\end{array}$ & $\begin{array}{l}\text { Grape fruit } \\
\text { juice }\end{array}$ & Quinine & None & $\begin{array}{c}\text { Torses de } \\
\text { pointes, convul- } \\
\text { sive Syncope } \\
\text { with Polydipsia. }\end{array}$ & $\begin{array}{l}\text { Flavonoid } \\
\text { naringen } \\
\text { increases } \\
\text { quinine } \\
\text { availability }\end{array}$ & $\begin{array}{c}\text { Likely } \\
1+1+1+0+1+ \\
1+1+1+1+0=8\end{array}$ \\
\hline $\begin{array}{l}113- \\
117 .\end{array}$ & $\begin{array}{c}5 \text { different cases, } \\
\text { no other } \\
\text { information }^{[127]}\end{array}$ & $\begin{array}{l}\text { Cranberry } \\
\text { juice }\end{array}$ & Warfarin & None & $\begin{array}{l}\text { Increase the } \\
\text { activity of war- } \\
\text { farin. }\end{array}$ & $\begin{array}{c}\text { Inhibit } \\
\text { breakdown of } \\
\text { warfarin in } \\
\text { body }\end{array}$ & $\begin{array}{l}\text { Unevaluable } \\
\begin{array}{c}0+1+0+0+0 \\
+0+0+1+0 \\
+0=2\end{array}\end{array}$ \\
\hline 118 & $\begin{array}{l}\text { A man in } 70 \text { 's } \\
\text { after a chest in- } \\
\text { fection and with a } \\
\text { poor appetite }{ }^{[128]}\end{array}$ & $\begin{array}{l}\text { Cranberry } \\
\text { juice }\end{array}$ & Warfarin & $\begin{array}{l}\text { Cefalexin } \\
\text { Digoxin } \\
\text { Phe- } \\
\text { nytoin }\end{array}$ & $\begin{array}{l}\text { Patient died of } \\
\text { GIT and pericar- } \\
\text { dial hemorrhage. }\end{array}$ & $\begin{array}{l}\text { Not de- } \\
\text { scribed }\end{array}$ & $\begin{array}{c}\text { Possible. } \\
\begin{array}{c}1+1+1+0+1 \\
+1+1+10+0 \\
=7\end{array}\end{array}$ \\
\hline $\begin{array}{l}119- \\
130\end{array}$ & $\begin{array}{l}12 \text { different cases } \\
\text { of interactions }{ }^{[129]}\end{array}$ & $\begin{array}{l}\text { Cranberry } \\
\text { juice }\end{array}$ & Warfarin & $\begin{array}{l}\text { Not de- } \\
\text { scribed }\end{array}$ & & $\begin{array}{l}\text { Not de- } \\
\text { scribed }\end{array}$ & $\begin{array}{c}\text { Unevaluable } \\
0\end{array}$ \\
\hline 131 & $\begin{array}{c}\text { A patient } \\
\text { No other } \\
\text { information }^{[130]}\end{array}$ & $\begin{array}{l}\text { Cranberry } \\
\text { juice }\end{array}$ & Warfarin & None & $\begin{array}{r}\text { High INR } \\
\text { Major bleedir }\end{array}$ & $\begin{array}{l}\text { Not de- } \\
\text { scribed }\end{array}$ & $\begin{array}{c}\text { Possible. } \\
0+0+0+1+0+1 \\
+0+1+1+0=4\end{array}$ \\
\hline 132. & $\begin{array}{l}\text { 70-year-old } \\
\text { women with } \\
\text { mechanical mitral } \\
\text { valve and } \\
\text { previous episode } \\
\text { of atrial } \\
\text { fibrillation. }{ }^{[131]}\end{array}$ & $\begin{array}{c}\text { Chamomile } \\
\text { (Matricaria } \\
\text { chamomilla) }\end{array}$ & Warfarin & $\begin{array}{l}\text { Amioda- } \\
\text { rone } \\
\text { Digoxin } \\
\text { Syn- } \\
\text { throid } \\
\text { Alendro- } \\
\text { nate } \\
\text { Metopro- }\end{array}$ & High INR (7.9) & $\begin{array}{l}\text { Coumarin } \\
\text { constituent } \\
\text { of herb } \\
\text { resulting in } \\
\text { supra thera- } \\
\text { peutic } \\
\text { anticoagula- } \\
\text { tion. }\end{array}$ & $\begin{array}{c}\text { Likely } \\
\begin{array}{c}1+1+1+1+1+1 \\
+1+1+0+0 \\
=09\end{array}\end{array}$ \\
\hline 133 & $\begin{array}{l}77 \text { year-old man } \\
\text { with history of } \\
\text { hypertension and } \\
\text { hyperuricemia. }{ }^{[132]}\end{array}$ & $\begin{array}{l}\text { Two Chinese } \\
\text { herbal medi- } \\
\text { cines, both } \\
\text { containing } \\
\text { glycyrrhizin. }\end{array}$ & & None & $\begin{array}{l}\text { Marked metabolic } \\
\text { alkalosis with } \\
\text { severe hypokale- } \\
\text { mia. Pseudoaldo- } \\
\text { steronism. }\end{array}$ & $\begin{array}{l}\text { Interaction of } \\
\text { rennin } \\
\text { angiotensin } \\
\text { system } \\
\text { with the } \\
\text { herb. }\end{array}$ & $\begin{array}{c}\text { Likely } \\
\begin{array}{c}1+1+0+1+ \\
1+1+1+1 \\
+1+0 \\
=08\end{array}\end{array}$ \\
\hline
\end{tabular}

The interfering drug may act as an inducer, inhibitor and / or substrate of the cytochrome P450 enzyme that is responsible for the metabolism of the respective drugs. This is the most important mechanism for interactions between herbal therapies and antiretroviral drugs. ${ }^{[19]}$ St. J ohn wort, widely prescribed for various psychopathologic conditions involving depression and anxiety ${ }^{[20]}$ is reported to lower serum concentrations of cyclosporine, ${ }^{[8,21-27]}$ theophyllin, ${ }^{[28]}$ warfarin, ${ }^{[29-30]}$ oral contraceptives ${ }^{[30]}$ digoxin, ${ }^{[31-33]}$ indinavir ${ }^{[34]}$ and clopidogrel ${ }^{[35]}$ by inducing CYP450 (CYP 3A4, CYP 2C9 and CYP1A2). ${ }^{[8,36-39]}$ On the other hand, the same herb has also been reported to inhibit the CYP $3 A 4$ isoform. ${ }^{[40]}$ Another mechanism stated for St. J ohn's wort, responsible for affecting clearance of many drugs is that it increases expression of duodenal P-glycoprotein (PgP), an ATP-dependent drug efflux transporter known to pump drugs out of the cell and thus decrease the intracellular concentration of drugs. ${ }^{[8,41-43]}$ Caution is warranted when using St. J ohn's wort with several medications like barbiturates, carbamazepine, dextromethorphan, fenfluramine, fexofenadine, narcotics, nortriptyline, phenytoin, photosensitizing drugs, reserpine and simvastatin during pregnancy and lactation for the risk of potential interactions. ${ }^{[44]}$

Pharmacodynamic interactions affect a drug's action in a qualitative way, either through enhancing effects (synergistic or additive actions) or antagonizing effects. The interactions reported in this category include those of St. John's wort with various antidepressants namely paroxetine, sertraline, nefazodone, venlafaxine, clonazepam and interactions of haloperidol ${ }^{[45-49]}$ with loperamide ${ }^{[50]}$ and kava-kava with alprazolam. ${ }^{[51]}$ Cases of serotonin syndrome (serotoninamplifying effect) with St. John's wort have also been studied. ${ }^{[52]}$ 
Ginkgo biloba, the secondmost common herb involved in drug interactions is reported to have potent drug interactions with fluoxetine, buspirone, insulin, monoamine oxidase inhibitors (MAOIs) and with drugs metabolized by cytochrome P450 3A4, P450 3A5, P450 1A2 and P450 2D6 enzymes. Hence, Ginkgo biloba should be used with caution during pregnancy and lactation. ${ }^{[53]}$ This antioxidant herb is mainly promoted for use in improving cognitive function (brain booster) and blood flow. ${ }^{[2,54]}$ It is reported to inhibit platelet aggregation activity resulting in spontaneous bleeding when used simultaneously with aspirin, ${ }^{[20,54]}$ acetaminophen, ${ }^{[20,54-55]}$ trazodone, ${ }^{[56]}$ ibuprofen ${ }^{[57]}$ and warfarin. ${ }^{[53-54,58]}$ Twenty-seven cases of ginkgo interactions were noted with acetaminophen, ibuprofen, nifedipine and sodium valproate in the present study, extending the list in a previous study where the herb was reported to interact with trazodone, warfarin, aspirin and thiazide diuretics. ${ }^{[8]}$ CYP2C9 is the CYP- 450 isoenzyme responsible for the metabolism of the more potent warfarin enantiomer, (S)-warfarin. Recently, a study conducted to ascertain the influence of Ginkgo biloba on CYP2C9, stated that Ginkgo biloba extract inhibits human liver microsomal CYP2C9 enzyme in vitro but not in vivo. ${ }^{[59]}$

Kava kava is shown to have additive effects with central nervous system depressants and caution is advised with regards to its simultaneous use with benzodiazepines, barbiturates, antipsychotics and alcohol. ${ }^{[20,51]}$ In addition, patients with Parkinson's disease are also discouraged from using kava products. ${ }^{[20,60]}$ In this report, eight new cases of kava-kava were listed with various drugs including levodopa, ${ }^{[60]}$ caffeine, ${ }^{[118]}$ hydrochlorthiazide, ${ }^{[119]}$ amino acid complex, ${ }^{[119]}$ promethazine and biperiden. ${ }^{[120]}$

Millions of people regularly take blood-thinning medications such as warfarin and over-the-counter drugs like aspirin and similar medications. These medications are very commonly prescribed for patients with high risk of blood clotting such as those with artificial heart valves, deep vein thrombosis or arterial fibrillation. The risk grows many fold when such patients take herbs possessing antiplatelet or anticoagulant activities and fail to report the use of herbs to their doctors. There are reports of potential herb-drug interactions with analgesic drugs, particularly aspirin, which have the potential to interact with herbal supplements that are known to possess antiplatelet activity as well as those containing coumarin components, thus enhancing the risk of bleeding. ${ }^{[61,62]}$ In the present study, warfarin was found to interact with at least 19 types of different herbs with a total of 34 cases of interactions adding to the list of 18 interactions previously reported ${ }^{[8]}$ There is a reasonable documentation of interactions between coumarin anticoagulants like warfarin with various herbs, ${ }^{[63]}$ apart from St J ohn's Wort, ginkgo biloba, ${ }^{[20,55,65]}$ panax ginseng, ${ }^{[20,64-65]}$ coenzyme- $Q,{ }^{[65]}$ siberian ginseng, ${ }^{[66]}$ danshen, ${ }^{[67-69]}$ dong quai, ${ }^{[70-72]}$ garlic, ${ }^{[73]}$ devil's claw, ${ }^{[74]}$ birch, ${ }^{[75]}$ boldo, fenugreek, ${ }^{[76]}$ green tea, ${ }^{[65,78]}$ quilinggao, a Chinese herbal product, ${ }^{[79]}$ Chinese herbal tea (Lycium barbarum) ${ }^{[80]}$ saw palmetto, ${ }^{[81-82]}$ papain, ${ }^{[121]}$ cranberry juice ${ }^{[127-130]}$ and chamomile. ${ }^{[131]}$ Taking much needed precautions such as regular monitoring of weekly prothrombin time (PT test) and International normalized ratio (INR) can prove to be helpful in avoiding risk due to these interactions.

Addition of herbal medicine to multiple drug therapy holds the potential for herb-drug interactions, ${ }^{[43,83-84]}$ although it is also suggested that some of the adverse drug effects and drug interactions reported for herbal products could be caused by impurities as allergens, pollen and spores or batch-tobatch variability. ${ }^{[20]}$ This is because unlike conventional drugs, herbal drug products are not regulated for their purity and potency. ${ }^{[20,21,85]}$

The present study also reports isolated cases of some herbs involved in interactions with various drugs such as ayahusca with fluoxetine, ${ }^{[86]}$ celery with thyroxin, ${ }^{[87]}$ fennel with enalapril,, ${ }^{[88]}$ prickly pear with an oral hypoglycemic agent, ${ }^{[89]}$ garlic with saquinavir, ${ }^{[90]}$ ginger with nonsteroidal anti-inflammatory drugs (NSAIDS), ${ }^{[91]}$ licorice with a laxative, ${ }^{\left[{ }^{[92-93]}\right.}$ soya bean with warfarin, ${ }^{[94]}$ alfalfa with immunosuppresants, ${ }^{[95]}$ shankhapushpi with phenytoin ${ }^{[96]}$ and chamomile with enalapril.[131] In most cases, the mechanism is unknown or just plain additive effects between the herbs and drugs responsible for the respective toxicity.

In an earlier report, concern was raised about herbdrug interactions with anesthesia and it was suggested that herbal therapies be discontinued at least two to three weeks prior to surgeries requiring anesthesia. ${ }^{[97-98]}$ Grapefruit juice too is reported to interact with number of drugs. Since the first report in 1989 as a result of using the juice as part of placebo preparation in a drug trial, ${ }^{[99-102]}$ the list has expanded tremendously. At present, grapefruit juice is reported to interact with number of anticancer agents including erlotinib, recently approved by the Canadian health authority as a monotherapy for locally advanced, small cell lung cancer. ${ }^{[103]}$

A broad concern is raised for a variety of potential interactions based on their pharmacological actions when case reports are not available. Examples are 1) laxative herbs (Aloe, licorice, senna, cascara) with potassium-wasting medications and with the drugs that act on the heart due to the risk of additional loss of potassium, ${ }^{[11,14,104-105]} 2$ ) herbs and drugs with similar actions like bitter melon and gymnema with other antidiabetic medications due to the risk of excessive hypoglycemia and 3 ) herbs like valerian, kava kava, ginger, goldenseal, chamomile with centrally acting agents due to the risk of increased sedation. Also worthy of mention are interactions of caffeine-containing herbs (Guarana, green tea) with other drugs that also affect the central nervous system ${ }^{[11,14,104]}$ and of herbs containing a coumarin component (black cohosh, chamomile, bilberry leaf) with warfarin due to the risk of hemorrhage. ${ }^{[14]}$ Recently, reports have emerged that the ayurvedic herbal medicine gugulipid or "guggul" may have unwanted interactions with many prescription medications. ${ }^{[106]}$ The herb is an Ayurvedic medicine taken to help lower cholesterol and stimulate thyroid function. It may interfere with drugs used for treating AIDS and cancer, specifically, gugulipid could interfere with drugs such as azathioprine, various other anticancer agents and cholesterol-lowering statins. The authors suggested that guggulsterone interacts with drugs by binding to a protein called pregnane $X$ receptor (PXR). PXRs induce the body to "turn on" a gene that encodes another protein that breaks down various drugs. Thus, the drug levels in the body are lowered. However, there are no case reports pertaining to interactions of guggul with any medication.

Inadequate reporting makes it very difficult to determine whether a particular herb-drug interaction has occurred. Proper 
documentation is necessary with all relevant information, clear description of adverse event and careful exploration of alternative explanations along with consideration for a reasonable re-challenge whenever possible.

Although, one or two reports may not warrant an absolute contraindication to combinations of herbal and prescription therapies, precautions do need to be exercised by taking the medical history of patients during counseling sessions to obtain this information. The onus lies on healthcare professionals who can monitor the use of herbal medicines, especially if the patients are taking them with prescription medications. An open-minded approach during patients' visits is to counsel the patients with enough information about signs and symptoms of herb-drug interactions so that they are able to recognize an adverse event if it occurs. It seems only rational that the administration of herb and drug, if taken together, should be at least 1 to 2 hours or several hours apart from each other. It is advisable to avoid using the herb and drug combinations altogether; which are contraindicated or reported frequently for interactions with the specific drug. Despite widespread use, herb-drug interactions are a stark reality today. The number of cases reported for the emerging herb-drug interactions are al ready on the rise. In fact, the actual number of cases may be higher due to under-reporting There are many plant compounds, which are still unfamiliar and new drugs are produced every day. Unfortunately, the herb-drug interaction remains an unknown area. It is now known that millions of patients take herbal and conventional medicines concomitantly, often without the knowledge of their physicians. Considering our present lack of understanding of herb-drug interactions, proper reporting of such cases, careful vigilance, evidence-based appraisal and constantly updated reviews of such information are very important to promote understanding in this area.

\section{References}

1. Mathur A. Who owns traditional knowledge? Working Paper No. 96, Indian Council for Research on International Economic Relations; 2003. p. 1-33

2. Agrawal OP, Raju PS. In: Abdin MZ, Abroi YP, editors. Global market of herbal products: Opportunities for India. Traditional system of medicine. Narosa Publishing House: New Delhi, India; 2006. p. 5-10.

3. Wakdikar S. Global health care challenge: Indian experiences and new prescriptions. Electronic J Biotechnol 2004;7:214-20.

4. Eisenberg DM, Davis RB, Ettner SL, Appel S, Wilkey S, Van Rompay M, et al. Trends in alternative medicine use in the United States, 1990-1997: Results of a follow-up national survey. JAMA 1998;28:1569-75.

5. Ramakrishnappa K. Biodiversity and the Ecosystem Approach in Agriculture, Forestry and Fisheries. New York, USA: 2003. Impact of Cultivation and Gathering of Medicinal Plants on Biodiversity: Case studies from India. United Nations Publications: p. 210-5.

6. WHO Traditional Medicine Strategy 2002-2005, Document WHO/EDM/ TRM/2002.1, WHO: Geneva; 2002. p. 11.

7. Fugh-Berman A. Herb-drug interactions. Lancet 2000;355:134-8.

8. Fugh-Berman A, Ernst E. Herb-drug interactions: Review and assessment of report reliability. Br J Clin Pharmacol 2001;52:587-95.

9. Winker MA, Flanagin A, Chi-Lum B, White J, Andrews K, Kennett RL, et al. Guidelines for medical and health information sites on the internet: Principles governing AMA web sites. American Medical Association. JAMA 2000;283:1600-6.

10. Wootton J. Directory of databases. The Vienna International Academy For Integrated medicine. The Rosenthal center for Complementary and Alternative medicine. 2004. [Last accessed on 2006 May 1]. Available from: http://cpmcnet. columbia.edu/dept/rosenthal/Databases.html.

11. Blumenthal M. Interactions between herbs and conventional drugs: Introductory considerations. Herbalgram 2000;49:52-63.
12. Jellin JM, Gregory PJ, Batz F, Hitchens K, Burson S, Shaver K, et al. Pharmacist's letter/Prescriber's letter Natural medicines comprehensive database. $5^{\text {th }}$ ed. Therapeutic Research Faculty: Stockton, CA; 2003. p. 1228-9.

13. Thompson Coon J, Pittler M, Ernst E. Herb-drug interactions: Survey of leading pharmaceutical/herbal companies. Arch Intern Med 2003;163:1371.

14. Lambrecht JE, Hamilton W, Rabinovich A. A review of herb-drug interactions: Documented and theoretical. US Pharma 2000;25:8.

15. Diane SG. Assessment of herb drug interaction in literature shows many case reports lack adequate information to determine report reliability. Herbalgram 2003; $57: 22-3$.

16. Izzo AA, Ernst E. Interactions between herbal medicines and prescribed drugs: A systematic review. Drugs 2001;61:2163-75.

17. Brazier N, Levine MAH. Drug-herb interaction among commonly used conventional medicines: A compendium for health care professionals. Am J Ther 2003;10:163-9.

18. Izzo AA. Drug interactions with St. John's Wort (Hypericum perforatum): A review of the clinical evidence. Int J Clin Pharmacol Ther 2004;42:139-48.

19. Rogers T. Herb-drug interactions. Canadian AIDS treatment information exchange (CATIE) 2004:01-06. [Last accessed on 2005 Jun 9]. Available from: http://www. catie.ca/pdf/supple-e/herb\%20drug\%20interactions.pdf.

20. Cupp MJ. Herbal remedies: Adverse effects and drug interactions. Am Fam Phy 1999;59:1239-45.

21. Barone GW, Gurley BJ, Ketel BL, Abul-Ezz Sr. Herbal supplements: A potential for drug interactions in transplant recipients. Transplantation 2001;71:239-41.

22. Karliova M, Treichel U, Malago M, Frilling A, Gerken G, Broelsch CE. Interaction of Hypericum perforatum (St. John's wort) with cyclosporin A metabolism in a patient after liver transplantation. J Hepatol 2000;33:853-5.

23. Mai I, Kruger H, Budde K, Johne A, Brockmoller J, Neumayer HH, et al. Hazardous pharmacokinetic interaction of Saint John's wort (Hypericum perforatum) with the immunosuppressant cyclosporin. Int J Clin Pharmacol Ther 2000;38:500-2.

24. Breidenbach TH, Hoffmann MW, Becker TH, Schlitt H, Klempnauer J. Drug interaction of St. John's wort with cyclosporin. Lancet 2000;355:1912.

25. Breidenbach T, Kliem V, Burg M, Radermacher J, Hoffmann MW, Klempnauer J. Profound drop of cyclosporin A whole blood trough levels caused by St. John's wort (Hypericum perforatum). Transplantation 2000;69:2229-30.

26. Ruschitzka F, Meier PJ, Turina M, Luscher TF, Noll G. Acute heart transplant rejection due to Saint John's wort. Lancet 2000;355:548-9.

27. Rey JM, Walter G. Hypericum perforatum (St John's wort) in depression: Pest or blessing? Med J Aust 1998;169:583-6.

28. Nebel A, Schneider BJ, Baker RA, Kroll DJ. Potential metabolic interaction between St. John's wort and theophylline. Ann Pharmacother 1999;33:502.

29. Yue QY, Bergquist C, Gerden B. Safety of St. John's wort (Hypericum perforatum). Lancet 2000;355:576-7.

30. Hall SD, Wang Z, Huang S, Mitchell A, Hamman BS, Vasavada N, et al. The interaction between St. John's wort and an oral contraceptive. Clin Pharmacol Ther 2003;74:525-35.

31. Cheng TO. St John's wort interaction with digoxin. Arch Intern Med 2000;160:2548.

32. Johne A, Brockmoller J, Bauer S, Maurer A, Langheinrich M, Roots I. Pharmacokinetic interaction of digoxin with an herbal extract from St John's wort (Hypericum perforatum). Clin Pharmacol Ther 1999;66:338-45.

33. Scott GN, Elmer GW. Update on natural product drug interactions. Am J Health Syst Pharm 2002;59:339-47.

34. Piscitelli SC, Burstein AH, Chaitt D, Alfaro RM, Falloon J. Indinavir concentrations and St. John's wort. Lancet 2000;355:547-8.

35. Lau WC, Carville DG, Guyer KE, Neer CJ, Bates ER. St. John's wort enhances the platelet inhibitory effect of clopidogrel in clopidogrel 'resistant' healthy volunteers. J Am Coll Cardiol 2005;45:382A.

36. Tatro DS, editor. Drug interaction facts: Herbal supplements and food. Saint Louis, MO. Facts and comparisons, ISBN 1574392123. A Walters Kluwer Company; 2004. Also available from: http://www.factsandcomparisons.com.

37. Roby CA, Anderson GD, Kantor E, Dryer DA, Burstein AH. St John's wort effect on CYP3A4 activity. Clin Pharmacol Ther 2000;67:451-7.

38. Kerb R, Bauer S, Brockmoller J, Roots I. Urinary- [hydroxyl cortisol excretion rate is affected by treatment with hypericum extract. Eur J Clin Pharmacol 1997;52:186

39. Cozza KL, Armstrong SC, Oesterheld JR, editors. Concise guide to drug interactions, Principles for medical practice, $2^{\text {nd }}$ ed. American Psychiatric Publishing: Washington DC; 2003. p. 11-40.

40. Obach RS. Inhibition of human cytochrome P450 enzymes by constituents of 
St. John's wort, an herbal preparation used in the treatment of depression. $J$ Pharmacol Exp Ther 2000;294:88-95.

41. Bressler R. Herb-drug interactions. St. John's wort and prescription medications. Geriatrics 2005;60:21-3.

42. Durr D, Stieger B, Kullak-Ublick GA, Rentsch KM, Steinert HC, Meier PJ, et al. St. John's wort induces intestinal P- glycoprotein/ MDR1 and intestinal and hepatic CYP3A4. Clin Pharmacol Ther 2000;68:598-604.

43. Yu DK. The contribution of P-glycoprotein to pharmacokinetic drug-drug interactions. J Clin Pharmacol 1999;39:1203-11.

44. Dugoua JJ, Mills E, Perri D, Koren G. Safety and efficacy of Ginkgo biloba during pregnancy and lactation. Can J Clin Pharmacol 2006;13:e277-84.

45. Waksman JC, Heard K, Joliff H, Daly FF, Bogdan GM, Dart RC. Serotonin syndrome associated with the use of St. John's wort and paroxetine. J Toxicol Clin Toxicol 2000;38:521.

46. Gordon JB. SSRls and St. John's wort: Possible toxicity? Am Fam Physician 1998; $57: 950,953$.

47. Barbanel DM, Yusufi B, O'Shea D, Bench CJ. Mania in a patient receiving testosterone replacement postorchidectomy taking St. John's wort and sertraline. J Psychopharmacol 2000;14:84-6.

48. Lantz MS, Buchalter E, Giambanco V. St. John's wort and antidepressant drug interactions in the elderly. J Geriatr Psychiatry Neurol 1999;12:7-10.

49. Prost N, Tichadou L, Rodor F, Nguyen N, David JM, Jean Pastor MJ. St. Johns wort-venlafaxine interaction. Presse Med 2000;29:1285-6.

50. Khawaja IS, Marotta RF, Lippmann S. Herbal medicines as a factor in delirium. Psychiatr Serv 1999;50:969-70.

51. Almeida JC, Grimsley EW. Coma from the health food store: Interaction between kava and alprazolam. Ann Intern Med 1996;125:940-1.

52. Cott JM, Fugh-Berman A. Is St. John's wort (Hypericum perforatum) an effective antidepressant? J Nerv Ment Dis 1998;186:500-1.

53. Dugoua JJ, Mills E, Perri D, Koren G. Safety and efficacy of Ginkgo biloba during pregnancy and lactation. Can J Clin Phamacol 2006;13:e277-84.

54. Rosenblatt M, Mindel J. Spontaneous hyphema associated with ingestion of Ginkgo biloba extract. N Engl J Med 1997;336:1108.

55. Rowin J, Lewis SL. Spontaneous bilateral subdural hematomas associated with chronic Ginkgo biloba ingestion. Neurology 1996;46:1775-6.

56. Galluzzi S, Zanetti O, Binetti G, Trabucchi M, Frisoni GB. Coma in a patient with Alzheimer's disease taking low dose trazodone and gingko biloba. J Neurol Neurosurg Psychiatry 2000;68:679-80.

57. Meisel C, Johne A, Roots I. Fatal intracerebral mass bleeding associated with Ginkgo biloba and ibuprofen. Atherosclerosis 2003;167:367.

58. Mathews MK. Association of Ginkgo biloba with intra cerebral hemorrhage. Neurology 1998:50:1933.

59. Mohutsky MA, Anderson GD, Miller JW, Elmer GW. Ginkgo biloba: Evaluation of CYP2C9 drug interactions in vitro and in vivo. Am J Ther 2006;13:24-31.

60. Schelosky L, Raffauf C, Jendroska K, Poewe W. Kava and dopamine antagonism. J Neurol Neurosurg Psychiatry 1995;58:639-40.

61. Abebe W. Herbal medication: Potential for adverse interactions with analgesic drugs. J Clin Pharm Ther 2002;27:391-401.

62. Dharmananda S. Checking for possible herb-drug interactions, PR. Institute for Traditional Medicine. Portland Oregon, 2003. [Last accessed on 2006 Mar 9]. Available from: http://www.itmonline.org/arts/herbdrug2.htm.

63. HeckAM, DeWitt BA, Luke AL. Potential interaction between alternative medicine and warfarin. Am J Health Syst Pharm 2000;57:1221-7.

64. Janetzky K, Morreale AP. Probable interaction between warfarin and ginseng. Am J Health Syst Pharm 1997;54:692-3.

65. Mandy CL. Interactions between Herbs and Cardiac Medications. Pharmacotherapy Update Newsletter, The Cleveland Clinic Center for ContinuingEducatio n.2001;IV [II]. [Last accessed on 2006 Mar 10]. Available from: http://www.clevelandclinicmeded.com/medical_info/pharmacy/MarApr2001/herbs_cardiac.htm.

66. McRae S. Elevated serum digoxin levels in a patient taking digoxin and siberian ginseng. CMAJ 1996;155:293-5.

67. Yu CM, Chan JC, Sanderson JE. Chinese herbs and warfarin potentiation by 'danshen'. J Intern Med 1997;241:337-9.

68. Izzat MB. A taste of Chinese medicine! Ann Thorac Surg 1998;66:941-2.

69. Tam LS, Chan TY, Leung WK, Critchley JA. Warfarin interactions with Chinese traditional medicines. Danshen and methyl-salicylate medicated oil. Aust NZ J Med 1995;25:258.

70. Ellis GR, Stephens MR. Untitled (Photograph and brief case report). In 'Minerva'. Br Med J 1999;319:650.

71. Page RL, Lawrence JD. Potentiation of warfarin by Dong Quai. Pharmacother
1999;19:870-6.

72. Wells PS, Holbrook AM, Crowther NR, Hirsh J. Interactions of warfarin with drugs and food. Ann Int Med 1994;131:676-83.

73. Sunter WH. Warfarin and garlic. Pharm J 1991;246:722.

74. Shaw D, Leon D, Kolev S, Murray V. Traditional remedies and food supplements: A 5-year toxicological study (1991-95). Drug Safety 1997;17:342-56.

75. Yip AS, Chow WH, Tai YT, Cheung KL. Adverse effect of topical methyl salicylate ointment on warfarin anticoagulation: An unrecognized potential hazard. Postgrad Med J 1990;66:367-9.

76. Lambert JP, Cormier A. Potential interaction between warfarin and boldo-fenugreek. Pharmacother 2001;21:509-12.

77. Smith M, Lin KM, Zheng YP. An open trial of nifedipine-herb interactions: Nifedipine with St. John's wort, ginseng or ginkgo biloba. Clin Pharmacol Ther 2001;69:86.

78. Taylor JR, Wilt VM. Probable antagonism of warfarin by green tea. Ann Pharmacother 1999;33:426-8.

79. Wong AL, Chan TY. Interaction between warfarin and the herbal product quilinggao. Ann Pharmacother 2003;37:836-8.

80. Lam AY, Elmer GW, Mohutsky MA. Possible interaction between warfarin and Lycium barbarum L. Ann Pharmacother 2001;35:1199-201.

81. Yue QY, Jansson K. Herbal drug Curbicin and anticoagulant effect with and without warfarin: Possibly related to the vitamin E component. J Am Geriatr Soc 2001;49:838.

82. Bressler R. Herb drug interactions: Interactions between saw palmetto and prescription medications. Geriatrics 2005;60:32-4.

83. Miller LG. Herbal medicines: Selected clinical considerations focusing on known or potential drug-herb interactions. Arch Intern Med 1998;158:2200-11.

84. Ernst E. The risk benefit profile of commonly used herbal therapies: Ginkgo, St. John's wort, Ginseng, Echinacea, Saw palmetto and Kava. Ann Intern Med 2002;136:42-53.

85. Angell M, Kassirer JP. Alternative medicine- The risks of untested and unregulated remedies. N Engl J Med 1998;339:839-41.

86. Callaway JC, Grob CS. Ayahuasca preparations and serotonin reuptake inhibitors: A potential combination for severe adverse interactions. J Psychoactive Drugs 1998;30:367-9.

87. Moses G. Thyroxine interacts with celery seed tablets? Aust Prescriber 2001;24:6-7.

88. Arya SC. Controlling angiotensin-converting-enzyme-inhibitor induced cough by fennel fruit. Indian J Pharmacol 1999;31:1999.

89. Meckes-Lozyoa M, Roman-Ramos R. Opuntia streptacantha: A coadjutor in the treatment of diabetes mellitus. Am J Chin Med 1986;14:116-8.

90. Piscitelli SC, Burstein AH, Welden N, Gallicano KD, Falloon J. The effect of garlic supplements on the pharmacokinetics of saquinavir. Clin Infect Dis 2002;34:234-8.

91. Srivastava KC, Mustafa T. Ginger (Zingiber officinale) and rheumatic disorders. Med Hypothesis 1989;29:25-8.

92. Brinker FJ. Online updates and additions to herb contraindications and drug interactions: $3^{\text {rd }}$ ed. Eclectic Medical Publications. [Last accessed on 2006 Feb 1]. Available from: http://www.eclecticherb.com/emp/updatesHCDI.html.

93. Ishiguchi T, Mikita N, Iwata T, Nakata H, Sato H, Higashimoto Y, et al. Myoclonus and metabolic alkalosis from licorice in antacid. Intern Med 2004;43:59-62.

94. Cambria-Kiely JA. Effect of Soy milk on warfarin efficacy. Ann Pharmacother 2002;36:1893-6.

95. Light TD, Light JA. Acute renal transplant rejection possibly related to herbal medications. Am J Transplant 2003;3:1608-9.

96. Dandekar UP, Chandra RS, Dalvi SS, Joshi MV, Gokhale PC, Sharma AV, et al. Analysis of clinically important interaction between phenytoin and shankhapushpi, an ayurvedic preparation. J Ethnopharmacol 1992;35:285-8.

97. Koupparis LS. Harmless herbs: A cause for concern. Anesthesia 2000;55:1012.

98. Dharmananda S. The interactions of herbs and drugs, Institute for Traditional Med, Portland Oregon. [Last accessed on 2000 March 9]. Available from: http://www. itmonline.org/arts/herbdrug.htm.

99. Bailey DG, Spence JD, Edgar B, Bayliff CD, Arnold JM. Ethanol enhances the hemodynamic effects of felodipine. Clin Invest Med 1989:12:357-62.

100. Bailey DG, Arnold JM, Spence JD. Grapefruit juice- drug interactions. Br J Clin Pharmacol 1998:46:101-10.

101. Fukuda K, Ohta T, Oshima Y, Ohashi N, Yoshikawa M, Yamazoe Y. Specific CYP3A4 inhibitors in grapefruit juice: Furocoumarin dimers as components of drug interaction. Pharmacogenetics 1997;7:391-6. 
102. Kane GC, Lipsky JJ. Drug-Grapefruit juice interactions. Mayo Clin Proc 2000;75:933-92

103. Kalyn R. Focus on erlotinib. British Columbia Cancer agency. Provin Syst Ther Update Prog 2006;9:1-3.

104. Jellin JM, editor. Natural medicine comprehensive database, Pharmacist's letter stockton. Therapeutic Research Faculty: 1999.

105. Elinov E, Chajek-Shaul T. Licorice consumption causing severe hypokalemic paralysis. Myo Clin Proc 2003;78:767-8.

106. Ding X, Staudinger JL. The ratio of constitutive androstane receptor to pregnane $X$ receptor determines the activity of guggulsterone against the Cyp2b10 promoter. J Pharmacol Exp Ther 2005;314:120-7.

107. Fung S. Drug interactions between select psychiatric herbal remedies. For your inpharmation. Pharma Newsletter 2001;21:15.

108. Crose S, McKeating K. Delayed emergence and St. John's wort. Anesthesiol 2002;96:1025-7.

109. Bolley R, Zulke C, Kammerl M, Fischereder M, Kramer BK. Tacrolimus-induced nephrotoxicity unmasked by induction of the CYP3A4 system with St. John's wort. Transplant 2002;73:1009.

110. Mathijssen RH, Verweij J, de Bruijn P, Loos WJ, Sparreboom A. Effects of St. John's wort on Irinotecan metabolism. J Nat Cancer Inst 2002;94:1247-9.

111. Granger AS. Ginkgo biloba precipitating epileptic seizures. Age Ageing 2001;30:523-5.

112. Fessenden JM, Wittenborn W, Clarke L. Ginkgo biloba: A case report of herbal medicine and bleeding postoperatively from a laparoscopic cholecystectomy. Am Surg 2001;67:33-5.

113. Ang-Lee MK, Moss J, Yuan CS. Herbal medicines and perioperative care. JAMA 2001;286:208-16.

114. Hopkins MP, Androff L, Benninghoff AS. Ginseng face cream and unexplained vaginal bleeding. Am J Obstet Gynecol 1988;159:1121-2.

115. Kabalak AA, Soyal OB, Urfalioglu A, Saracoglu F, Gogus N. Menometrorrhagia and tachyarrhythmia after using oral and topical ginseng. J Womens Health (Larchmt) 2004;13:830-3.

116. Gonzalez-Seijo JC, Ramos YM, Lastra I. Maniac episode and ginseng: Report of a possible case. J Clin Psychopharmacol 1995;15:447-8.

117. Vazquez I, Aguera-Ortiz LF. Herbal products and serious side effects: A case of ginseng- induced manic episode. Acta Psychiatr Scand 2002;105:76-8.

118. Donadio V, Bonsi P, Zele I, Monari L, Liguori R, Vetrugno R, et al. Myoglobinuria after ingestion of extracts of Guarana, Ginkgo biloba and kava. Neurol Sci 2000;21:124.

119. Schmidt M, Harsewinkel, Nahrstedt A. Is Kava really hepatotoxic? An analysis of the known data on adverse effect of kava preparations on the liver. The German Federal Inst for Drugs and Medical Devises.bfArM-No.98004297 CRC letter. July 2002. [Last accessed on 2006 Mar 10]. Available from: http://www.munichre. net/publications/CRC_letter_2002_07_en.pdf?rdm=84496.

120. Gruenwald J, Mueller C, Skrabal J. Kava report 2003. In depth investigation in to EU member states market restrictions on kava products, part II-A: Expert report on clinical documentation on kava kava (Piper methysticum), Center for development of Entreprise (CDE). Brussels, Belgium, March 2003. [Last accessed on 2006 Mar 10]. Available from: http://background.de/Papers/KavaReport2003Part_IIA.pdf.

121. Pérez-Jáuregui J, Escate-Cavero A, Vega-Galina J, Ruiz-Arguelles GJ, MacipNieto $\mathrm{G}$. A probable case of wafarin overdose during anti-inflammatory therapy. Rev Invest Clin 1995;47:311-3.

122. Joss JD, LeBlond RF. Potentiation of warfarin anticoagulation associated with topical methyl salicylate. Ann Pharmacother 2000;34:729-33.

123. Russmann S, Winkler A, Lovblad KO, Stanga Z, Bassetti C. Lethal Ischemic Stroke after Cisplatin-based chemotherapy for testicular carcinoma and cannabis inhalation. Eur Neurol 2002;48:178-80.

124. Naik SD, Freudenberger RS. Ephedra-associated cardiomyopathy. Ann Pharmacother 2004;38:400-3.

125. Dreier JP, Endres M. Statin-associated rhabdomyolysis triggered by grapefruit consumption. Neurology 2004;62:670.

126. Hermans K, Stockman D, Van den Branden F. Grapefruit and tonic: A deadly combination in a patient with the long QT syndrome. Am J Med 2003;114:511-2.

127. Lee TH. By the way, doctor. I've been on warfarin (Coumadin) since 1979 after having a Starr-Edwards valve put in. The cardiologist insists on aggressive therapy and wants my INR at 3.5. But l've had several serious bleeds in the past year and was hospitalized three times. What's the current thinking about adequate INR levels for patients with metal heart valves? Harv Health Lett 2003;29:8.

128. Suvarna R, Pirmohamed M, Henderson L. Possible interaction between warfarin and cranberry juice. BMJ 2003;327:1454.

129. Committee on Safety of Medicines. Possible interaction between warfarin and cranberry juice. Curr Prob Pharmacovigilance 2004;30:10.

130. Rindone JP, Murphy TW. Warfarin-cranberry juice interaction resulting in profound hypoprothrombinemia and bleeding. Am J Ther 2006;13:283-4.

131. Segal R, Pilote L. Warfarin interactions with Matricaria chamomilla. CMAJ 2006;174:1281-2.

132. lida R, Otsuka Y, Matsumoto K, Kuriyama S, Hosoya T. Pseudoaldosteronism due to the concurrent use of two herbal medicines containing glycyrrhizin with angiotensin-converting enzyme inhibitor. Clin Exp Nephrol 2006;10:131-5.

\section{Author Help: Online Submission of the Manuscripts}

Articles can be submitted online from http://www.journalonweb.com. For online submission articles should be prepared in two files (first page file and article file). Images should be submitted separately.

\section{1) First Page File:}

Prepare the title page, covering letter, acknowledgement, etc., using a word processor program. All information which can reveal your identity should be here. Use text/rtf/doc/pdf files. Do not zip the files.

\section{2) Article file:}

The main text of the article, beginning from Abstract till References (including tables) should be in this file. Do not include any information (such as acknowledgement, your names in page headers, etc.) in this file. Use text/rtf/doc/pdf files. Do not zip the files. Limit the file size to $400 \mathrm{~kb}$. Do not incorporate images in the file. If file size is large, graphs can be submitted as images separately without incorporating them in the article file to reduce the size of the file.

3) Images:

Submit good quality colour images. Each image should be less than $\mathbf{4 0 0} \mathbf{~ k b}$ in size. Size of the image can be reduced by decreasing the actual height and width of the images (keep up to about 4 inches) or by reducing the quality of image. All image formats (jpeg, tiff, gif, bmp, png, eps, etc.) are acceptable; jpeg is most suitable. The image quality should be good enough to judge the scientific value of the image. Alw ays retain a good quality, high resolution image for print purpose. This high resolution image should be sent to the editorial office at the time of sending a revised article.

4) Legends:

Legends for the figures/images should be included at the end of the article file. 